\title{
Pollution et risque alimentaire dans les jardins partagés franciliens
}

Pollution and food safety in the community gardens of Paris metropolitan region

\section{Laurence Baudelet}

\section{(2) OpenEdition}

\section{Journals}

Édition électronique

URL : http://journals.openedition.org/ethnoecologie/2441

DOI : $10.4000 /$ ethnoecologie. 2441

ISSN : 2267-2419

Éditeur

Laboratoire Eco-anthropologie et Ethnobiologie

Référence électronique

Laurence Baudelet, "Pollution et risque alimentaire dans les jardins partagés franciliens », Revue d'ethnoécologie [En ligne], 8 | 2015, mis en ligne le 31 décembre 2015, consulté le 19 avril 2019. URL : http://journals.openedition.org/ethnoecologie/2441 ; DOI : 10.4000/ethnoecologie.2441

Ce document a été généré automatiquement le 19 avril 2019

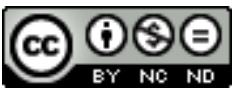

Revue d'ethnoécologie est mis à disposition selon les termes de la licence Creative Commons Attribution - Pas d'Utilisation Commerciale - Pas de Modification 4.0 International. 


\title{
Pollution et risque alimentaire dans les jardins partagés franciliens ${ }^{1}$
}

Pollution and food safety in the community gardens of Paris metropolitan region

\author{
Laurence Baudelet
}

1 Le retour d'une production horticole dans les villes françaises s'est opéré au tournant des années 2000, y compris dans Paris, avec le déploiement de jardins collectifs sous gestion associative et l'installation plus récente de petites unités de production à vocation commerciale. Les premières initiatives de création « jardins collectifs » datent, en France, du milieu des années 1990 (Dubost \& Lizet 2003). Depuis lors ces dernières n'ont cessé d'augmenter. Elles se comptent aujourd'hui par centaines. Nous désignons sous le vocable de "jardins collectifs», les différentes formes de jardinage impliquant un groupe de jardiniers à l'œuvre sur un espace dédié à cette pratique. Nous y incluons les jardins familiaux, jardins partagés, jardins d'insertion, pédagogiques ou encore les jardins à vocation thérapeutique ainsi que les actions de fleurissement participatif dans l'espace public et privé, le guerilla gardening ou encore les vergers collectifs. Il existe de nombreuses déclinaisons locales de ces typologies qui s'hybrident bien souvent sur le terrain. Ces dénominations par «famille» demeurent précieuses cependant car elles permettent de caractériser la vocation principale d'un projet de jardinage.

\section{Entre désir et inquiétude : la pollution en question}

2 L'importance de ce mouvement à l'échelle nationale s'accompagne d'une préoccupation grandissante concernant la qualité sanitaire des plantes alimentaires qui poussent en milieu urbain, du fait de la présence supposée ou avérée de polluants dans le sol, l'eau et l'air. L'inquiétude ressurgit régulièrement dans les médias qui ne manquent pas de relayer ce qu'ils considèrent comme un enjeu de santé dont le consommateur citadin doit être informé. D'invisible, au sens propre et au figuré, la pollution urbaine et ses effets sur la santé humaine sont devenus de plus en plus visibles dans le débat public. La 
consommation de végétaux cultivés dans des jardins urbains n'est qu'une sous-partie d'une question plus vaste.

3 Dans notre pratique professionnelle, nous notons que ce questionnement autour de la pollution est aujourd'hui partagé par les citoyen/ne/s, les services techniques comme par les élu/e/s des collectivités locales et d'une manière générale par les différents acteurs engagés dans des projets de jardinage collectif. Cela ne signifie pas pour autant que l'ensemble de ces acteurs soit mobilisé sur la question de la pollution dans chaque projet de jardin. Localement il peut y avoir des disparités dans l'appropriation de cet enjeu, certains acteurs y étant plus sensibles que d'autres. Cette disparité génère parfois des tensions, mais a le mérite de poser les problèmes. Nous y reviendrons plus en détail dans la suite de cet article.

4 La communauté scientifique en France commence également à s'intéresser de près à la question des risques de contamination des plantes cultivées dans les jardins urbains associatifs. C'est un fait récent car jusqu'à présent nous disposions de peu d'études sur ce sujet. Ainsi le programme national de recherche intitulé JASSUR ${ }^{2}$ (Jardins ASSociatifs URbains et villes durables) qui a débuté en 2012, comporte-t-il un volet dédié à « une caractérisation bio-physico-chimique des sols et des produits issus des jardins»en relation avec la question de la pollution. Les résultats seront communiqués à partir de 2016, à l'issue des trois années de recherche. Ce programme est co-dirigé au niveau national par Christophe Schwartz, pionnier des études sur les sols de jardins (Schwartz 2013). Dans la même période 2012-2015, l'Agence d'Écologie Urbaine, rattachée à la Direction des Espaces Verts et de l'Environnement (DEVE) de la Ville de Paris, a lancé une étude baptisée POTagers Expérimentaux (POTEX) sur 4 sites présentant des situations contrastées d'exposition à des polluants potentiellement présents dans le sol, l'eau ou l'atmosphère. Pour mener à bien cette étude, la division Impact Santé Environnement de l'Agence d'Écologie Urbaine, s'est adjoint un comité scientifique constitué de l'Institut National de l'Environnement Industriel et des Risques (INERIS), du Centre d'Études et d'Expertise sur les Risques, l'Environnement, la Mobilité et l'Aménagement (CEREMA) et de l'École Nationale d'Agronomie de Toulouse. L'Agence Régionale de Santé (ARS) d'Îlede-France a été associée au projet. Des liens existent entre les équipes des deux études précitées, qui ont fait une présentation commune de leurs résultats provisoires en juin $2015^{3}$.

5 Les Éléments Traces Métalliques (ETM), aussi appelés «métaux lourds », tels que le plomb, le cadmium, le cuivre ou le zinc, font l'objet d'une attention particulière des pouvoirs publics. Ils sont en partie bioassimilables par les plantes et présentent des risques pour la santé humaine qu'en cas de fortes concentrations dans le sol. Une base de données nationale appelée BAPPET ${ }^{4}$ (Base de données des teneurs en éléments traces métalliques de plantes potagères) regroupe depuis 2007 toutes les informations scientifiques concernant la contamination des plantes potagères par les ETM. La base est téléchargeable sur le site du Ministère de l'Écologie qui souhaite ainsi en faciliter l'accès au plus grand nombre.

6 Les mêmes préoccupations se font jour au niveau européen. L'étude menée à Berlin par l'Université Technique sur les taux de concentration en métaux lourds dans les fruits et légumes produits dans la capitale allemande, a eu un large écho dans les médias français lors de sa publication en 2012 (Säumel et al. 2012). Les résultats concernant les sites les plus exposés à la pollution liée au trafic routier font apparaître des taux de concentration supérieurs aux normes sanitaires européennes. Ce n'est pas surprenant, mais le fait que 
des mesures scientifiques le démontrent a fait alors prendre conscience à des collectivités de la nocivité de certains sites. Nombre de jardins familiaux sont en effet installés en contrebas d'axes routiers très fréquentés.

7 Paradoxalement, du moins en apparence, le terme d'agriculture urbaine, fait florès. On ne compte plus les colloques qui lui sont consacrés ni les projets de toute nature qui se font jour, chaque année plus nombreux. L'agriculture urbaine fait désormais l'objet de la commande publique. Des parcs urbains intègrent des exploitations maraîchères comme le parc départemental des Lilas à Vitry-sur-Seine (Val-de-Marne) ou des jardins associatifs comme le parc des Docks à Saint-Ouen (Seine-Saint-Denis) inauguré en 2013, qui comporte en outre des ruches et une serre de $1400 \mathrm{~m}^{2}$. Dans la feuille de route ${ }^{5}$ adressée en mai 2014 à son adjointe en charge des Espaces Verts, Anne Hidalgo, nouvellement élue maire de Paris, lui demande de contribuer au "développement collaboratif » de l'agriculture urbaine dans la capitale. Et pour cela de consacrer 30 hectares de toituresterrasses à cette fonction mais aussi de réserver des espaces pour les jardins partagés dans les nouveaux squares.

8 Le déploiement d'activités de production agricole en milieu urbain s'accompagne souvent d'une démarche expérimentale qui situe ces activités dans le champ de l'ingénierie bioclimatique, bien loin semble-t-il, des questions de pollution. Or ce n'est pas le cas, les porteurs de projet lient les différents enjeux. L'appel à projet "Végétalisation innovante » ${ }^{6}$ lancé en 2013 par la Ville de Paris en collaboration avec Paris Région Lab en est la parfaite illustration. Il s'inscrit à la fois dans le Plan Biodiversité et dans le Plan Climat Énergie parisiens. Et il compte deux projets ${ }^{7}$ de phytoépuration de l'eau et de l'air parmi les 30 lauréats. Dans leur quasi-totalité, les projets retenus ont recours à des dispositifs de production hors-sol. Nous voyons ainsi apparaitre une agriculture urbaine reposant sur des techniques de culture sophistiquées qui s'affranchissent des sols, contournant dès lors la question de leur éventuelle pollution et de leur pauvreté en nutriments. Il demeure cependant le problème de la pollution atmosphérique. L'T̂le-de-France y reste particulièrement soumise même si la tendance sur le long terme est à la baisse. Les seuils de pollution autorisés y sont régulièrement dépassés comme l'indiquent les rapports annuels d'Airparif, en particulier pour le dioxyde d'azote, l'ozone et les particules fines. Les zones les plus exposées se trouvent dans Paris, les départements de la Petite Couronne, les zones urbaines de la Grande Couronne et les grands axes routiers ${ }^{8}$. Ces données sont aujourd'hui disponibles en tant réel sur le site d'Airparif et donc connues du grand public.

9 La culture en toitures-terrasses pourrait concilier le modèle vertueux d'une agriculture urbaine respectueuse de l'environnement, pourvoyeuse de denrées saines, voire de qualité biologique, avec un milieu pollué. Elle semble moins exposée aux effets de la pollution aérienne. Le potager expérimental créé sur la toiture d'AgroParisTech en 2011, témoigne de concentrations en Éléments Traces Métalliques inférieures aux normes françaises et européennes dans l'ensemble des cultures (Grard et al. 2014). Et le même projet, baptisé T4P, démontre qu'il est possible de produire un substrat de qualité à partir de déchets organiques abondants en ville tels que le marc de café ou les déchets verts.

10 L'enjeu principal en matière de pollution se concentre donc sur la pleine terre. Les surfaces concernées sont plus importantes et les risques de contamination également. Les activités polluantes d'un passé industriel récent se sont inscrites dans les sols urbains et dans nos mémoires collectives. Les habitants qui désirent jardiner connaissent, pour les plus anciens, l'histoire de leur quartier et les emplacements potentiellement pollués. Ils 
en font état lors des réunions, questionnent les élus et s'interrogent sur les risques induits par la consommation des végétaux cultivés. En outre, l'île-de-France, aujourd'hui très associée dans les représentations au secteur tertiaire, conserve en réalité une activité industrielle qui peut présenter à la fois des risques de pollution et d'accidents technologiques jusqu'au cœur de l'agglomération. Trente-neuf sites sont actuellement classés SEVESO seuil haut par la Direction régionale et interdépartementale de l'Environnement et de l'Énergie (DRIEE) dont sept se trouvent très près de Paris, comme les dépôts d'hydrocarbure de Gennevilliers, Nanterre ou Vitry-sur-Seine'.

11 En somme, l'articulation entre les données concernant la pollution des milieux franciliens et la demande sociale nouvelle qui s'exprime en faveur d'une agriculture la plus «écologique » possible et de proximité, dans laquelle j'inclus les jardins collectifs sous toutes leurs formes, n'est donc pas chose aisée. La thèse récente de Jeanne Pourias (2014) sur la fonction alimentaire des jardins associatifs urbains parisiens et montréalais apporte un éclairage intéressant. Son étude concerne des jardins familiaux et des jardins partagés de tailles différentes. La plus petite parcelle cultivée est de $6 \mathrm{~m}^{2}$ et la plus grande de $500 \mathrm{~m}^{2}$. Sur un échantillon de 14 jardiniers franciliens, 10 déclarent couvrir entre 50 et $100 \%$ de leurs besoins en produits frais durant la saison de culture et l'un des jardiniers se dit complètement autosuffisant en végétaux toute l'année du fait de sa production et de la transformation des produits du jardin en conserves. Or selon Jeanne Pourias, les dires des jardiniers sont cohérents avec les quantités produites et mesurées pour chacun d'entre eux. La dimension d'autoconsommation n'est donc pas négligeable. Elle a déjà été soulignée et étudiée (Cérézuelle \& Roustand 2010) plus spécifiquement pour ce qui concerne les jardins familiaux ${ }^{10}$.

12 En nous appuyant sur les exemples du programme Main Verte parisien et sur le jardin partagé du quartier de Gournay-les-Usines à Creil dans l'Oise, examinons maintenant les stratégies à l'œuvre sur le terrain pour évaluer le risque sanitaire de ces cultures, la prise en charge du coût d'étude et éventuellement de dépollution, ainsi que la façon dont le risque est assumé par les habitants et par les collectivités ou les bailleurs sociaux qui mettent du foncier à disposition des projets de jardinage ou de maraîchage.

\section{Entre principe de précaution et relation subjective au territoire}

13 Nous avons pu observer jusqu'à présent trois stratégies concernant les risques de pollution dans les projets de jardins partagés : la "politique de l'autruche », l'application systématique du principe de précaution et la co-construction de réponses adaptées.

14 La "politique de l'autruche » consiste pour le propriétaire du foncier à proposer des terrains à cultiver sans réaliser d'analyses de sol ni de recherches précises sur l'occupation antérieure du site. La question de la pollution est ainsi évincée et disparait des débats à moins qu'elle ne soit réintroduite par les habitants. C'est bien sûr une façon d'éviter les mauvaises surprises et les surcoûts. Si nous prenons en considération l'ensemble des projets de jardins partagés que nous avons suivis en Île-de-France depuis 2001, date de la création de Graine de Jardins, nous constatons que cette position est devenue minoritaire. La question de la pollution est abordée de plus en plus en amont du projet par les élus et les services des collectivités ou par les bailleurs sociaux mais aussi par les habitants. Parfois le risque de pollution n'est pas évoqué car il est jugé nul, à juste 
titre. C'est un autre cas de figure qui suppose une connaissance de l'historique des activités.

L'application du principe de précaution est en quelque sorte le revers de la même médaille. Cette fois le sol est présumé pollué et le propriétaire prend toutes les garanties en imposant notamment de cultiver les plantes comestibles dans des contenants remplis de terre végétale pour éviter tout risque. La convention d'occupation du terrain peut aussi stipuler que la consommation des végétaux se fait sous la seule responsabilité du jardinier. Les choix d'aménagement opérés au titre du programme Main Verte rattaché à la Direction des Espaces Verts et de l'Environnement de Paris (DEVE), illustrent cette deuxième stratégie avec des nuances. La Ville fait en effet systématiquement une étude historique pour tout projet de jardin partagé occupant une friche lui appartenant. Si l'étude ne révèle aucun risque, les cultures peuvent se faire en pleine terre mais depuis 2003, seuls trois sites ont pu en bénéficier. Si les risques sont jugés « modérés ", seules les parties du jardin dédiées aux plantes alimentaires sont aménagées en «hors-sol »; les plantes ornementales peuvent être cultivées en pleine terre et une partie du terrain peut même demeurer en friche pour enrichir la biodiversité. Le « hors-sol » se traduit par un décaissement de la couche supérieure du sol, la pose d'un géotextile ${ }^{11}$, d'une couche drainante et un apport de terre végétale. C'est un procédé assez coûteux que la Ville de Paris essaie maintenant de limiter aux parties du terrain contaminées. Quant au plan annexé à la convention d'occupation du terrain, il en précise les usages, en accord avec l'association signataire. La situation est différente pour les jardins partagés situés dans des parcs publics, en création ou en cours de rénovation. Les sols ne sont jamais conservés. La DEVE rapporte de la terre végétale et ajoute un géotextile dans la partie occupée par le jardin partagé. Je qualifierais de " différenciée » cette gestion du risque en fonction des usages. Si certains habitants-jardiniers ne sont pas toujours convaincus de l'utilité de faire des analyses de sol, Karina Prevost, la responsable de Main Verte, souligne qu'ils sont toujours curieux d'en connaitre les résultats.

16 La dernière des stratégies observées est celle de bailleurs sociaux ou de collectivités locales confrontés à un problème de pollution avéré sur un terrain à cultiver. $\mathrm{Ne}$ disposant pas de protocole pré-établi, ils doivent trouver des solutions à la fois techniques et budgétaires. Il arrive de plus en plus que la solution soit co-construite avec le groupe de jardiniers. Ils sont informés et associés à la réflexion, voire à l'aménagement du terrain. Ces débats nécessitent de faire effort de pédagogie. Intervient ici la question de l'acceptabilité sociale de la pollution. En effet, les seuils fixés par les agences publiques ne se superposent pas avec nos représentations. L'arsenic par exemple renvoie à l'idée d'un poison très toxique pour la santé humaine. Même à des doses inférieures aux normes, sa présence nous parait incompatible avec toute production comestible.

17 Un autre paramètre à prendre en compte est celui du rapport intime de chacun des jardiniers au territoire et à sa mémoire. Le projet de jardin partagé porté par la Communauté d'Agglomération Creilloise (CAC) à Gournay-les-Usines est intéressant de ce point de vue. Nous y avons travaillé de 2010 à 2012. Le terrain choisi pour accueillir temporairement le jardin partagé avait vu se succéder de nombreuses activités industrielles et il était fortement contaminé par des métaux lourds. Le quartier de Gournay possède encore des usines de métallurgie dont une importante aciérie. Le problème de la pollution des sols a été évoqué dès la première réunion par les représentants de la CAC. Les taux étant très élevés, le sol devait être confiné pour que les 
jardiniers ne ramènent pas chez eux une terre contaminée, susceptible d'être ingérée par des enfants.

18 La réaction des habitants a été attentive et sereine. À aucun moment ils n'ont remis en cause le projet. Tout au contraire, ils ont participé de manière très active à la « réhabilitation du terrain » pour le transformer en «jardin écologique». La solution la plus économique a consisté en un épandage de broyat de palettes sur toute la parcelle. Des contenants en textile (Bacsacs) ont ensuite été disposés et remplis de terre végétale par les habitants avant d'être plantés. Le groupe a également creusé une grande mare. On peut faire l'hypothèse que leur rapport quotidien à un environnement industriel et le fait qu'ils aient travaillé dans ces usines pour certains, ont construit un rapport familier avec les risques liés à la pollution. Cette mise en scène du vivant (plantes cultivées, mare) a contribué à la réhabilitation symbolique du terrain et bien sûr du quartier.

Figure 1 : Aménagement du jardin des Marais (Creil), automne 2011

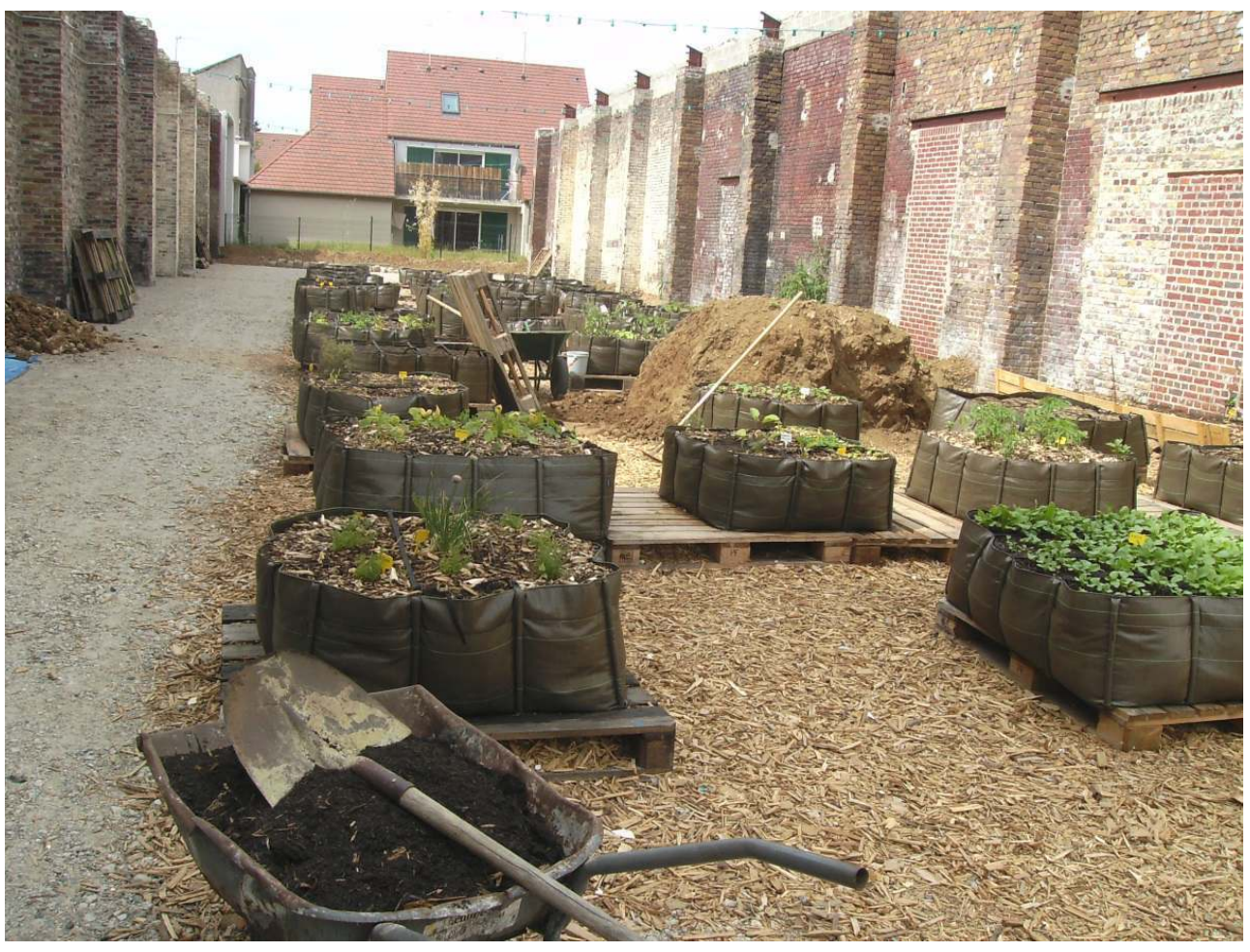

(c) GRAINE DE JARDINS 
Figure 2 : Le jardin des Marais (Creil), juillet 2012

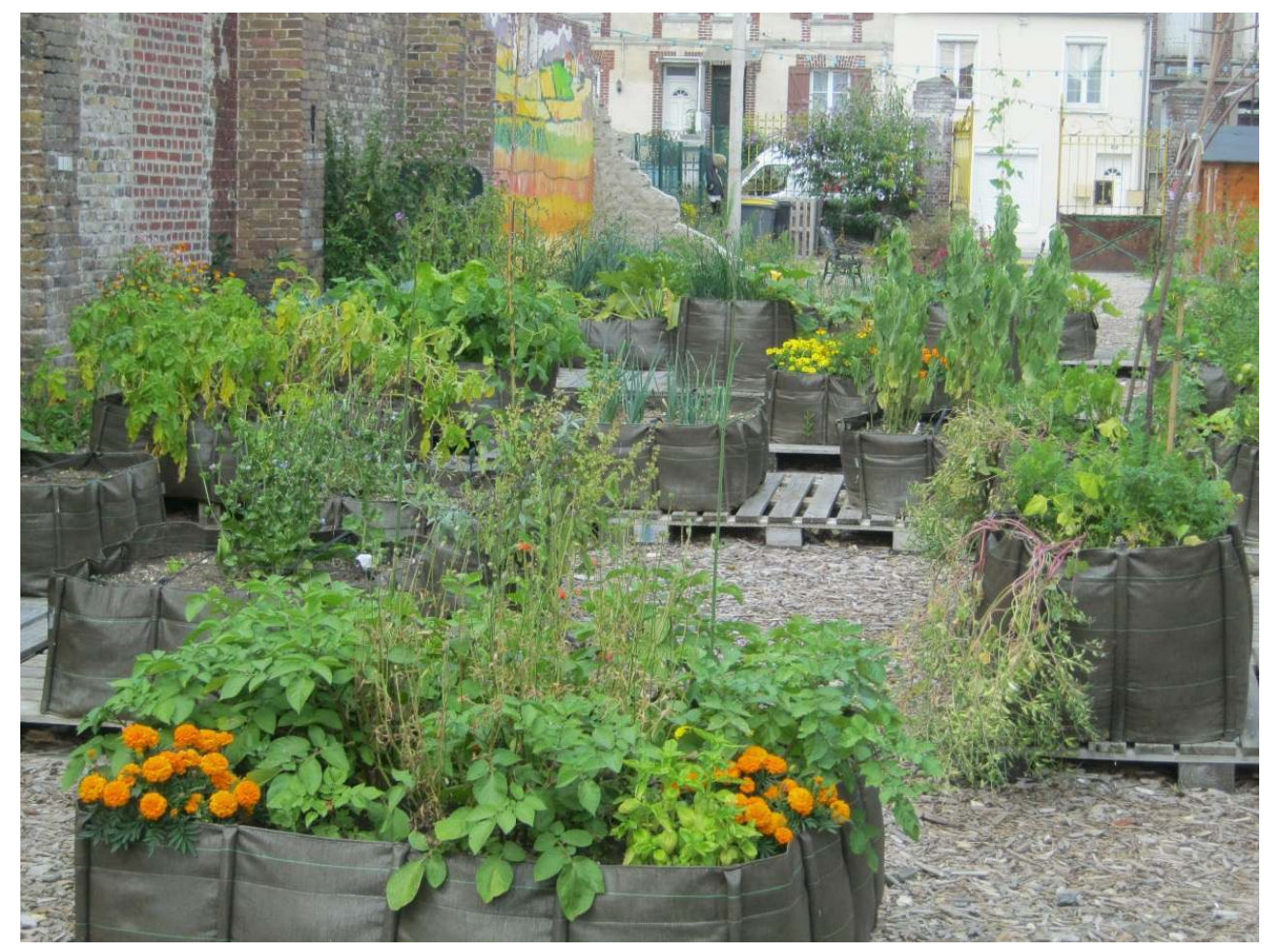

(c) GRAINE DE JARDINS

\section{L'inversion de la charge}

19 Il est intéressant d'observer que jusque dans les années 2000, ce sont les jardiniers amateurs qui étaient considérés comme les principaux responsables de la contamination de leurs végétaux. L'attention portait moins sur la pollution présente dans l'environnement que sur celle générée par les pratiques culturales. Les jardiniers avaient en effet la réputation, sans doute non usurpée pour nombre d'entre eux, d'avoir « la main lourde » sur les engrais de synthèse pour avoir de beaux et gros légumes mais aussi sur les phytosanitaires afin d'éradiquer les pestes pouvant diminuer les récoltes. Les quelques études réalisées à l'époque étaient assez inquiétantes. Les associations de jardinage familial se trouvaient particulièrement montrées du doigt. Ces jardiniers ne faisaient pourtant que suivre la pente d'une agriculture française industrialisée, première consommatrice de phytosanitaires à l'échelle européenne.

20 La société civile s'est emparée de la question avant les pouvoirs publics. L'association pour le Mouvement pour les Droits et le Respect des Générations Futures a commencé à alerter l'opinion et les responsables politiques dès 1996. Parmi les jeunes associations de jardinage, le réseau national des jardins partagés, le Jardin dans Tous Ses États, a fondé sa Charte (1997) sur des pratiques de jardinage respectueuses de l'environnement. Mais il faudra attendre une dizaine d'années avant que les ministères de l'Environnement et de l'Agriculture ne lancent le Plan Écophyto adressé aux agriculteurs professionnels et aux non professionnels. L'objectif pour les non professionnels, dont font partie les associations de jardinage, était de réduire de $50 \%$ «si possible" l'utilisation des phytosanitaires sur dix ans, de 2008 à 2018. N'ayant qu'une valeur déclarative, ce Plan n'a 
cependant pas permis d'atteindre ses objectifs (Potier 2014). Il a malgré tout fortement mobilisé les associations de jardinage familial et la Société Nationale d'Horticulture de France sur ce sujet. Ces structures ont en effet depuis évolué dans leur discours vers un jardinage raisonné, privilégiant des méthodes alternatives détaillées sur le site internet ${ }^{12}$ créé dans le cadre du Plan Écophyto. Dans le même temps beaucoup de collectivités locales ont commencé à limiter l'utilisation des pesticides dans l'entretien des espaces verts, voire à les supprimer pour passer en « zéro-phyto ». La loi Labbé ${ }^{13}$ " visant à mieux encadrer l'utilisation des produits phytosanitaires" votée en 2014 et renforcée tout récemment par la loi sur la "transition énergétique ", interdit d'ailleurs aux collectivités d'utiliser ces produits, sauf à titre exceptionnel, dès 2017. Les jardiniers amateurs ne pourront quant à eux plus y avoir recours à partir de 2019. Les chartes de jardinage collectif des grandes villes interdisent également le recours aux pesticides depuis plusieurs années.

Cela ne signifie pas que les membres des associations de jardinage respectent tous ces prescriptions à la lettre et ont renoncé à l'emploi de ces produits. Des conflits existent au sein des associations entre les tenants des méthodes « chimiques» et ceux qui veulent faire du « bio ». C'est une question de génération aussi. Dans nos missions de terrain, nous constatons une évolution très nette du discours des urbains qui s'intéressent au jardinage mais n'en ont aucune expérience. Leur souhait est de cultiver de la manière la moins polluante pour l'environnement et la moins dangereuse pour la santé. Force est de constater que les nouveaux jardiniers urbains sont majoritairement des femmes, qui semblent plus concernées par les enjeux de santé et d'alimentation que leurs homologues masculins.

\section{Conclusion}

L'utopie de la ville jardinée, nourricière dans ses différentes strates, recyclant ses déchets, se heurte à une connaissance de plus en plus précise des polluants présents dans le sol, l'air et l'eau. Les technologies bio-intensives en hors-sol apportent en partie réponse à ce défi. Leur développement à large échelle dépendra de la maîtrise des coûts en investissement et en fonctionnement des unités de production, dans un temps où les collectivités locales font face à des baisses importantes des dotations de l'état et où le modèle économique de ces activités agricoles n'est pas encore assis. Il existe cependant d'autres solutions que le hors-sol pour rendre nos terres urbaines fertiles. La phytoremédiation en constitue un exemple. Elle s'inscrit dans un temps long et permet de conserver des terrains non artificialisés, précieux puits de carbone à l'heure du dérèglement climatique. L'agriculture urbaine contribuerait ainsi d'autant mieux à la résilience des villes (Boukharaeva \& Marloie 2011). On pourrait enfin élargir le débat en se demandant s'il existe des milieux non pollués, en s'interrogeant sur l'origine des sols importés, ou encore en remettant en question la qualité sanitaire et gustative de notre alimentation issue d'une agriculture industrielle. 


\section{BIBLIOGRAPHIE}

Boukharaeva L. \& Marloie M. 2011 - Des sols agricoles au service de la résilience urbaine : réflexions à partir du cas de la Russie. Espaces et Sociétés 147 : 155-172.

Cérézuelle D. \& Roustand G. 2010 - Autoproduction accompagnée. Un levier de changement. Toulouse, ERES, 208 p. (Sociologie économique).

Dubost F. \& Lizet B. 2003 - La nature dans la cité. Communications Numéro spécial sous la direction de Françoise Dubost et Bernadette Lizet « Bienfaisante nature » 74 : 5-18. DOI: 10.3406/ comm.2003.2125

Grard B., Bel N., Marchal N., Madre F., Cambier P., Castell J.F., Manoucheri N., Besançon S., Houot S., Frascaria Lacoste N., Chenu C. \& Aubry C. 2014 - Cultiver sur les toits? Premiers résultats de l'expérimentation T4P (Toit Parisien Productif, Projet Pilote) à Paris, France. Colloque « Nourritures jardinières dans les sociétés urbanisées », Centre Culturel International de Cerisy, Aout 2014. Potier D. 2014 - Pesticides et agro-écologie, les champs du possible. Rapport pour le Premier Ministre. http://agriculture.gouv.fr/ministere/pesticides-et-agro-ecologie-les-champs-du-possible Pourias J. 2014 - Production alimentaire et pratiques culturales en agriculture urbaine. Analyse agronomique de la fonction alimentaire des jardins associatifs urbains à Paris et Montréal. Thèse de doctorat, Institut des Sciences et Industries du Vivant et de l'Environnement (AgroParisTech). [En ligne] : http://www.jardinons-ensemble.org/IMG/pdf/these_jpourias_2014_finale.pdf Säumel I., Kotsyuk I, Hoelscher M. Lenkereit C., Weber F. \& Kowarik I. 2012 - How healthy is urban horticulture in high traffic areas? Trace metal concentrations in vegetable crops from plantings within inner city neighbourhoods in Berlin, Germany. Environmental Pollution 165 : 124-132. DOI: 10.1016/j.envpol. 2012.02.019

Schwartz C. 2013 - Les sols de jardins, supports d'une agriculture urbaine intensive, Vertigo, Horssérie 15. https://vertigo.revues.org/12858\#tocto2n2

\section{NOTES}

1. Cet article s'appuie sur mon expérience d'accompagnement de projet de jardins partagés en région francilienne depuis 2001 et sur les réflexions menées en parallèle avec différentes équipes de recherche.

2. JASSUR : http://www.agence-nationale-recherche.fr/?Projet=ANR-12-VBDU-0011

3. Atelier Ville de Paris/JASSUR, Les Jardins collectifs en milieu urbain, responsabilités et espaces publics, 11 juin 2015, Pavillon du Lac, parc de Bercy (Paris).

4. BAPPET : http://www.developpement-durable.gouv.fr/BAPPET-BAse-de-donnees-sur-les.html

5. Feuille de route de Colombe Brossel, mai 2014. http://labs.paris.fr/commun/adjoints/ BROSSEL.pdf

6. Appel à projet Végétalisation innovante: www.parisregionlab.com/projet/112

7. http://expe.parisregionlab.com/actu/130

8. Surveillance et qualité de l'air en Île-de-France en 2014, www.airparif.asso.fr/_pdf/ publications/bilan-2014.pdf 
9. Liste des établissements SEVESO seuil haut en Île-de-France, DRIEE IDF. www.driee.ile-defrance.developpement-durable.gouv.fr/IMG/pdf/Liste_AS_corrigee_cle27d332.pdf

10. Loi $n^{\circ}$ 52-895 du 26 juillet 1952, Art. 1 Sont considérés comme « jardins familiaux » au regard de la présente loi, les parcelles de terre que leurs exploitants cultivent personnellement, en vue de subvenir aux besoins de leur foyer, à l'exclusion de tout usage commercial.

11. Membrane perméable en matière synthétique ou naturelle (en jute et fibre de coco) qui empêche les polluants présents dans le sol de contaminer les végétaux cultivés dans la terre posée sur ce feutre.

12. http://www.jardiner-autrement.fr/

13. Loi $n^{\circ} 2014-110$ du 6 février 2014.

\section{RÉSUMÉS}

Le retour d'une production horticole dans les villes françaises s'accompagne d'une préoccupation grandissante concernant la qualité sanitaire des plantes alimentaires qui poussent en milieu urbain. La consommation des produits des cultures par les jardiniers, loin d'être négligeable, est mise en débat du fait de la présence supposée ou avérée de polluants dans le sol, l'eau et l'air. La « politique de l'autruche » n'est plus à l'ordre du jour et usagers des jardins, services techniques, élus et communauté scientifique se saisissent aujourd'hui vigoureusement de la question. De l'application systématique du principe de précaution par les pouvoirs publics à la coconstruction de réponses adaptées, il s'agit en effet aujourd'hui de faire face au problème. Dans ce cadre, l'enjeu principal se concentre sur la pleine terre et le recours à des dispositifs de production hors-sol constitue la réponse la plus fréquente au problème, même si demeure alors celui de la pollution atmosphérique. La phytoremédiation, quant à elle, ne rencontre pas un grand succès. Pourtant, elle s'inscrit dans un temps long et permettrait de conserver des terrains non artificialisés, précieux puits de carbone à l'heure du dérèglement climatique.

The return of horticultural production in French cities is accompanied by a growing concern about the health quality of food plants. The consumption of crops by gardeners, far from insignificant, draws attention because of the supposed or actual presence of pollutants in soil, water and air. Burying one's head in the sand is no longer on the agenda. On the contrary, municipalities tend to systematically apply the precautionary principle. The main focus is nowadays on polluted soils and off-ground cultivation solutions constitute the most common answer to address pollution issues, even if the atmospheric context remains critical. Meanwhile phytoremediation has little success. Yet, wouldn't it be a longer-term solution, preserving nonartificialised land and offering valuable urban carbon sink when climate change is at stake.

\section{INDEX}

Mots-clés : jardins collectifs, pollution, hors-sol, stratégies d'acteurs, débat public

Keywords : collective gardening, off-ground cultivation, actors' strategies, public debate 


\section{AUTEUR}

\section{LAURENCE BAUDELET}

Association Graine de Jardins - Paris 